\title{
First CUORE-0 Performance Results and Status of CUORE Experiment
}

L. Canonica - D. R. Artusa - F. T. Avignone III - O. Azzolini - M. Balata •

T. I. Banks - G. Bari - J. Beeman - F. Bellini - A. Bersani - M. Biassoni •

T. Bloxham - C. Brofferio - C. Bucci - X. Z. Cai - A. Camacho • X. Cao •

S. Capelli - L. Carbone - L. Cardani - M. Carrettoni - N. Casali - D. Chiesa -

N. Chott - M. Clemenza - C. Cosmelli - O. Cremonesi - R. J. Creswick •

I. Dafinei • A. Dally • V. Datskov • A. De Biasi • M. M. Deninno •

S. Di Domizio - M. L. di Vacri • L. Ejzak - R. Faccini - D. Q. Fang •

H. A. Farach - M. Faverzani - G. Fernandes - E. Ferri - F. Ferroni •

E. Fiorini - M. A. Franceschi - S. J. Freedman - B. K. Fujikawa •

A. Giachero - L. Gironi - A. Giuliani - J. Goett - P. Gorla - C. Gotti •

T. D. Gutierrez · E. E. Haller • K. Han • K. M. Heeger • H. Z. Huang •

R. Kadel • K. Kazkaz • G. Keppel • L. Kogler • Yu. G. Kolomensky •

D. Lenz • Y. L. Li - C. Ligi - X. Liu - Y. G. Ma • C. Maiano • M. Maino •

M. Martinez - R. H. Maruyama - Y. Mei • N. Moggi • S. Morganti •

T. Napolitano - S. Newman - S. Nisi - C. Nones - E. B. Norman •

A. Nucciotti - T. O'Donnell - F. Orio - D. Orlandi - J. L. Ouellet •

M. Pallavicini - V. Palmieri - L. Pattavina - M. Pavan - M. Pedretti •

G. Pessina - G. Piperno - C. Pira - S. Pirro - E. Previtali - V. Rampazzo •

F. Rimondi - C. Rosenfeld • C. Rusconi - E. Sala - S. Sangiorgio •

N. D. Scielzo - M. Sisti - A. R. Smith - L. Taffarello - M. Tenconi •

F. Terranova - W. D. Tian - C. Tomei - S. Trentalange - G. Ventura •

M. Vignati - B. S. Wang • H. W. Wang • L. Wielgus • J. Wilson • T. Wise •

A. Woodcraft · L. Zanotti - C. Zarra - B. X. Zhu - S. Zucchelli

Received: 30 July 2013 / Accepted: 15 January 2014 / Published online: 11 February 2014

(C) Springer Science+Business Media New York 2014

Abstract The CUORE (Cryogenic Underground Observatory for Rare Events) experiment will search for neutrinoless double beta decay in ${ }^{130} \mathrm{Te}$. Observation of the process would unambiguously establish that neutrinos are Majorana particles as well as provide information about the absolute neutrino mass scale and mass hierarchy.The CUORE setup will consist of an array of 988 tellurium dioxide crystals (containing

S. J. Freedman and F. Rimondi-Deceased.

L. Canonica $(\bowtie) \cdot$ D. R. Artusa - M. Balata - T. I. Banks - C. Bucci · N. Casali · M. L. di Vacri ·

J. Goett · P. Gorla · S. Newman · S. Nisi · D. Orlandi · L. Pattavina · C. Zarra

INFN - Laboratori Nazionali del Gran Sasso, Assergi, AQ, Italy

e-mail: lucia.canonica@lngs.infn.it 
$206 \mathrm{~kg}$ of ${ }^{130} \mathrm{Te}$ in total), operated as bolometers at a temperature of $\sim 10 \mathrm{mK}$. The experiment is now under construction at the Gran Sasso National Laboratory in Italy. As a first step towards CUORE, a tower (CUORE-0) has been assembled and is taking data. Here a detailed description of the CUORE-0 tower and its performance is reported. The status of the CUORE experiment and its expected sensitivity will then be discussed.

D. R. Artusa · F. T. Avignone III - N. Chott · R. J. Creswick · H. A. Farach · S. Newman · C. Rosenfeld · J. Wilson

Department of Physics and Astronomy, University of South Carolina, Columbia, SC 29208, USA

O. Azzolini · A. Camacho · A. De Biasi · G. Keppel · V. Palmieri · C. Pira · V. Rampazzo

INFN - Laboratori Nazionali di Legnaro, 35020 Padua, Legnaro, Italy

T. I. Banks · S. J. Freedman · L. Kogler · Yu. G. Kolomensky · T. O’Donnell · J. L. Ouellet Department of Physics, University of California, Berkeley, CA 94720, USA

T. I. Banks · T. Bloxham · S. J. Freedman · B. K. Fujikawa · K. Han · L. Kogler · Y. Mei · J. L. Ouellet Nuclear Science Division, Lawrence Berkeley National Laboratory, Berkeley, CA 94720, USA

G. Bari · M. M. Deninno · N. Moggi · F. Rimondi · S. Zucchelli

INFN - Sezione di Bologna, 40127 Bologna, Italy

J. Beeman · E. E. Haller

Materials Science Division, Lawrence Berkeley National Laboratory, Berkeley, CA 94720, USA

F. Bellini · L. Cardani · C. Cosmelli · R. Faccini · F. Ferroni ·

G. Piperno

Dipartimento di Fisica, Sapienza Università di Roma, 00185 Rome, Italy

F. Bellini · L. Cardani · C. Cosmelli · I. Dafinei - R. Faccini · F. Ferroni - S. Morganti · F. Orio · G. Piperno $\cdot$ C. Tomei $\cdot$ M. Vignati

INFN - Sezione di Roma, 00185 Rome, Italy

A. Bersani · S. Di Domizio · G. Fernandes · M. Pallavicini

INFN - Sezione di Genova, 16146 Genoa, Italy

M. Biassoni - C. Brofferio - S. Capelli - M. Carrettoni - D. Chiesa - M. Clemenza ·

M. Faverzani · E. Ferri - E. Fiorini - A. Giachero - L. Gironi · C. Gotti ·

C. Maiano - M. Maino - A. Nucciotti - M. Pavan - E. Sala - M. Sisti · L. Zanotti

Dipartimento di Fisica, Università di Milano-Bicocca, 20126 Milan, Italy

M. Biassoni - C. Brofferio - S. Capelli - L. Carbone · M. Carrettoni · D. Chiesa - M. Clemenza ·

O. Cremonesi - V. Datskov · M. Faverzani - E. Ferri · E. Fiorini · A. Giachero · L. Gironi · C. Gotti ·

C. Maiano - M. Maino - A. Nucciotti - M. Pavan - G. Pessina - S. Pirro - E. Previtali - C. Rusconi ·

E. Sala $\cdot$ M. Sisti $\cdot$ F. Terranova $\cdot$ L. Zanotti

INFN - Sezione di Milano Bicocca, 20126 Milan, Italy

X. Z. Cai · X. Cao · D. Q. Fang · Y. L. Li · Y. G. Ma · W. D. Tian · H. W. Wang

Shanghai Institute of Applied Physics (Chinese Academy of Sciences), Shanghai 201800, China

A. Dally · L. Ejzak · K. M. Heeger · D. Lenz · R. H. Maruyama · L. Wielgus · T. Wise

Department of Physics, University of Wisconsin, Madison, WI 53706, USA 
S. Di Domizio · G. Fernandes · M. Pallavicini

Dipartimento di Fisica, Università di Genova, 16146 Genova, Italy

M. A. Franceschi · C. Ligi - T. Napolitano

INFN - Laboratori Nazionali di Frascati, 00044 Frascati, Rome, Italy

A. Giuliani - M. Tenconi

Centre de Spectrométrie Nucléaire et de Spectrométrie de Masse, 91405 Orsay, France

T. D. Gutierrez

Physics Department, California Polytechnic State University, San Luis Obispo, CA 93407, USA

E. E. Haller

Department of Materials Science and Engineering, University of California, Berkeley, CA 94720, USA

H. Z. Huang · X. Liu · S. Trentalange · B. X. Zhu

Department of Physics and Astronomy, University of California, Los Angeles, CA 90095, USA

R. Kadel · Yu. G. Kolomensky

Physics Division, Lawrence Berkeley National Laboratory, Berkeley, CA 94720, USA

K. Kazkaz · E. B. Norman · M. Pedretti · S. Sangiorgio · N. D. Scielzo · B. S. Wang

Lawrence Livermore National Laboratory, Livermore, CA 94550, USA

\section{Martinez}

Laboratorio de Fisica Nuclear y Astroparticulas, Universidad de Zaragoza, 50009 Zaragoza, Spain

C. Nones

Service de Physique des Particules, CEA / Saclay, 91191 Gif-sur-Yvette, France

E. B. Norman · B. S. Wang

Department of Nuclear Engineering, University of California, Berkeley, CA 94720, USA

F. Rimondi · S. Zucchelli

Dipartimento di Fisica, Università di Bologna, 40127 Bologna, Italy

A. R. Smith

EH\&S Division, Lawrence Berkeley National Laboratory, Berkeley, CA 94720, USA

L. Taffarello

INFN - Sezione di Padova, 35131 Padua, Italy

G. Ventura

Dipartimento di Fisica, Università di Firenze, 50125 Florence, Italy

G. Ventura

INFN - Sezione di Firenze, 50125 Florence, Italy

A. Woodcraft

SUPA, Institute for Astronomy, University of Edinburgh, Blackford Hill, Edinburgh EH9 3HJ, UK

Present Address:

J. Goett

Los Alamos National Laboratory, Los Alamos, NM 87544, USA 
Keywords Neutrinoless double beta decay · Neutrino mass - Majorana particle ·

Bolometer

\section{Introduction: $\mathrm{TeO}_{2}$ Bolometers for Rare-Event Searches}

Neutrinoless double-beta decay (0vDBD) is a rare nuclear transition not allowed in the Standard Model framework. Its discovery would indicate the Majorana nature of neutrinos and provide information on the hierarchy of the neutrino masses and scales [1].

The purpose of the CUORE experiment is to search for OvDBD in ${ }^{130} \mathrm{Te}$ using $\mathrm{TeO}_{2}$ crystals operated as cryogenic bolometers. The 0vDBD Q-value for this nucleus is $2527.5 \mathrm{keV}$ [2]. The CUORE detector will consist of a tightly packed array of 988 $\mathrm{TeO}_{2}$ crystals containing $206 \mathrm{~kg}$ of ${ }^{130} \mathrm{Te}$ and cooled inside a large cryostat operated at $10 \mathrm{mK}$. At this low temperature the crystals work as highly sensitive calorimeters, converting the energy deposited by particle interactions into measurable temperature rises.

Each $\mathrm{TeO}_{2}$ crystal, $5 \times 5 \times 5 \mathrm{~cm}^{3}$ with a mass of $750 \mathrm{~g}$, serves as both the source of double-beta decaying ${ }^{130} \mathrm{Te}$ and the energy absorber. The crystals are mechanically and thermally coupled to a copper holder, acting as heat sink, using small Teflon (PTFE) pieces. A Neutron Transmutation Doped Ge thermistor (NTD), for the detection of temperature variations, and a heater, are glued on each crystal. The heaters are used to inject pulses of heat into the crystals at regular intervals, to stabilize the bolometer response against small fluctuations in operating temperature over time. $\mathrm{TeO}_{2}$ bolometers have long been used to search for 0vDBD in ${ }^{130}$ Te because their properties are well matched to the experimental requirements [3,4]. Taking into account the large-mass of the experiment, this determines a high sensitivity to OvDBD, which makes CUORE one of the most competitive experiments in the field.

A prototype of CUORE, Cuoricino, took data at Gran Sasso National Laboratories in the years 2003-2008. Besides demonstrating the feasibility of a large mass bolometric detector, Cuoricino set the most stringent half-life limit for the neutrinoless double-beta decay of ${ }^{130} \mathrm{Te}:>2.8 \times 10^{24}$ year at $90 \%$ C.L., with a corresponding upper bound on the neutrino Majorana mass in the range $(0.30$ 0.71) $\mathrm{eV}$ [5]. Cuoricino was also a precious tool for the understanding of the key problematics to be solved for the construction of CUORE. An intermediate step has preceded the start of CUORE: its first tower, named CUORE-0, is being operated in the former Cuoricino cryostat and is now acquiring data. CUORE-0 served as a test of the new CUORE assembly line, as well as a high-statistics check of the improvements implemented to reduce the background sources and to improve the bolometric response of the detectors. It will also be a promising detector, that will surpass the limit on the effective neutrino mass set by Cuoricino.

Present Address:

L. Kogler

Sandia National Laboratories, Livermore, CA 94551, USA 


\section{The CUORE Experimental Challenges}

The aim of future neutrinoless double beta decay experiments like CUORE is to probe the inverted hierarchy region of neutrino masses. Reaching such a challenging sensitivity requires very demanding specifications on the design and construction of the CUORE detector. The key experimental parameters for achieving this goal are: extremely low radioactive background, excellent detector energy resolution and long live time of the measurement.

To accomplish background suppression, the first step was identification of the main sources responsible for Cuoricino background in the Region Of Interest (ROI), whose measured value was of $(0.169 \pm 0.006)$ counts $/ \mathrm{keV} / \mathrm{kg} / \mathrm{y}$. By means of Monte Carlo simulations [6] two main background sources were identified: surface contaminations of the detector components (responsible of $\sim 60 \%$ of the ROI background), and ${ }^{232} \mathrm{Th}$ bulk contaminations of the materials surrounding the experimental set up (e.g. cryostat), mainly due to ${ }^{208} \mathrm{Tl}$ decays (responsible of $\sim 30 \%$ of the ROI background). Surface contaminations of detector parts (mainly the copper structure surfaces facing the absorber and the crystal surfaces themselves) are the most dangerous because they produced degraded alphas, that release only part of their energy in the crystal absorber. These events can contribute to a flat background that goes from the full alpha energy peak to much lower energies, reaching the neutrinoless double beta decay ROI. The main contaminants were identified as coming from the ${ }^{238} \mathrm{U}$ and ${ }^{232} \mathrm{Th}$ natural decay chains.

The knowledge gained from Cuoricino guided the design and construction of CUORE. After a series of thorough studies on the background abatement, a special detector structure was designed to reduce the copper surface area facing the detectors. New surfaces-cleaning techniques were defined to reduce surface contaminations and minimize the induced background in the ROI [7], [8]. Moreover, all radiochemically pure materials are continuously kept under strict controls to prevent possible recontaminations. To cool the CUORE detector a large cryogen-free cryostat with five pulse tubes and one specially designed high-power dilution refrigerator has been designed. The detector assembly has a total mass of about 1.5 tonne and uses a vibration decoupling suspension system. Because of the stringent radioactivity requirements, about 10 tonnes of lead shielding will need to be cooled below $4 \mathrm{~K}$, and only a limited number of construction materials are acceptable.

\section{CUORE-0}

The final step before the start of the CUORE experiment is CUORE-0, a single CUORE-like tower that is now acquiring data in the former Cuoricino cryostat. CUORE- 0 consists of 52 CUORE crystals with a total $\mathrm{TeO}_{2}$ mass of $39 \mathrm{~kg}$. It has been assembled accordingly to the same stringent protocols defined for CUORE. CUORE-0 represents an opportunity to evaluate the bolometric performances of a CUORE-like detector apparatus in a familiar cryostat, and it will be the first large-scale empirical test of the extensive background-reduction measures undertaken. 


\subsection{CUORE-0 Assembly}

Tower construction is organized around two units: the gluing station, which provides all the tools needed for the gluing of thermal sensors onto the crystals, followed by the assembly line, an integrated set of tools devoted to the final assembly of the tower. Both units use $\mathrm{N}_{2}$-fluxed glove boxes to provide a controlled radon-free environment [9]. The coupling of the sensors (thermistors and heaters) to the $\mathrm{TeO}_{2}$ crystal absorbers affects the performance of the detector. Significant effort went into improving the totally manual gluing procedure used previously in Cuoricino and obtain a highly reproducible sensor-to-absorber coupling procedure. The sensor-to-absorber coupling is made by applying a matrix of glue dots using a semiautomated system involving robots which allows high repeatability.

The CUORE-0 tower has been assembled following strict protocols under extremely clean conditions. This not only requires that all assembly be performed inside gloveboxes flushed with nitrogen gas, but also that strict controls are implemented on all materials which come into contact with the tower components during assembly. The assembly line consists of five separate glove boxes for specialized operations on the detector components. The assembly includes also the system and procedures for the electrical connection of the sensors to the tower wires, consisting of PEN (Polyethylene naphthalate) strips with a copper deposition forming the wires. The connection is achieved by bonding four 25-micron wires from the sensor gold pads to the copper pads on the wire strips. During the bonding of the CUORE-0 tower connections, we were able to connect 51 out of 52 NTD thermistors and 51 out of 52 heater sensors.

\subsection{Performances}

The CUORE-0 detector was cooled down in September 2012. Unfortunately, due to problems with the old Cuoricino cryostat, the detector has achieved stable operation only since March 2013. During the first cool down we lost one electrical connection of one heater chip, reducing the number of active heaters to 50 out of 52 and bringing to 49 the number of fully active channels (crystals equipped with both working heater and NTD chips). However, we verified that no thermometer connections were lost during the multiple cool-downs, meaning that the new bonding system can easily tolerate thermal contractions.

After we reached base temperature of about $10 \mathrm{mK}$ on all the detectors, we performed the optimization of the bolometers. Load curves were computed to determine the best configuration of the thermistor bias circuit and to have maximum pulse amplitudes. To evaluate the bolometric performances of the detectors we performed a calibration measurement, by inserting a gamma source (thoriated tungsten wires) outside of the cryostat. Figure 1 shows the energy spectrum of pulse amplitudes recorded by 49 fully active channels with the calibration sources in place. The statistics was acquired in $258.7 \mathrm{~h}$. The peaks in Fig. 1 are identified as gamma lines from the decay of nuclei in the ${ }^{232}$ Th decay chain.

The energy resolution is evaluated on the $2615 \mathrm{keV}$ photo-peak from the decay of ${ }^{208} \mathrm{Tl}$, very close to the ${ }^{130} \mathrm{Te} \mathrm{Q}$-value of $2,527.5 \mathrm{keV}$. Fig. 2 shows the gaussian fit 


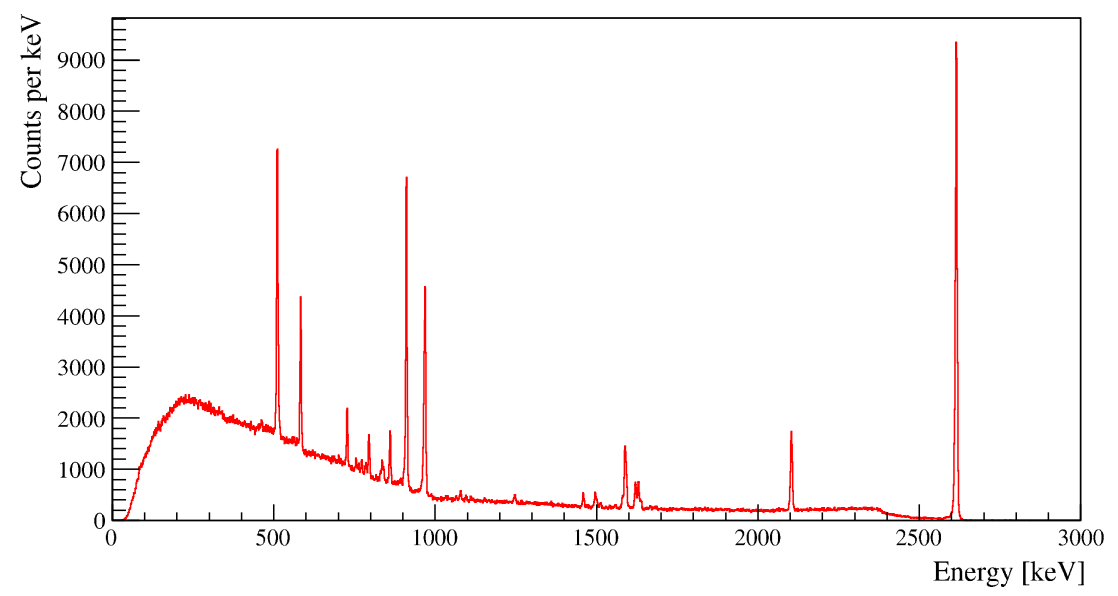

Fig. 1 The sum spectrum of the calibration run from 49 fully active channels of the CUORE-0 detector. The visible lines are due to $\gamma \mathrm{s}$ from the ${ }^{232}$ Th calibration source (Color figure online)

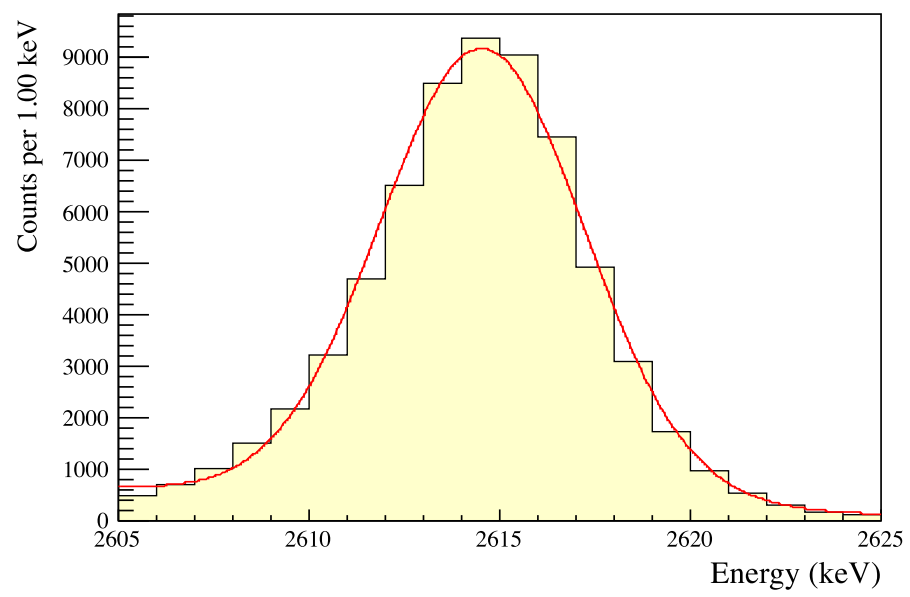

Fig. 2 Fit of the $2615 \mathrm{keV}{ }^{208} \mathrm{Tl} \gamma$ peak in the sum spectrum of the calibration runs from the 49 fully active channels of the CUORE-0 detector (Color figure online)

superimposed on a linear flat background to the $2,615 \mathrm{keV}$ calibration peak for the sum spectrum of all the 49 fully active channels; the energy resolution is $(6.3 \pm 2.7) \mathrm{keV}$. For several months CUORE-0 has been acquiring background data.

CUORE-0 is operating in the cryostat used for Cuoricino and consequently the $\gamma$ background from contamination in the cryostat shields will remain approximately the same as in Cuoricino. Considering that the irreducible background for CUORE-0 comes from the $2615 \mathrm{keV}^{208} \mathrm{Tl}$ line due to ${ }^{232} \mathrm{Th}$ contaminations in the cryostat, in the case that all other background sources (e.g. surface contaminations) will be negligible, this would imply a lower limit of 0.05 counts $/ \mathrm{keV} / \mathrm{kg} / \mathrm{y}$ on the expected background.

Similarly, a conservative upper limit of 0.11 counts $/ \mathrm{keV} / \mathrm{kg} / \mathrm{y}$ follows from scaling the Cuoricino background by a factor of 2, proven to be at reach in dedicated 


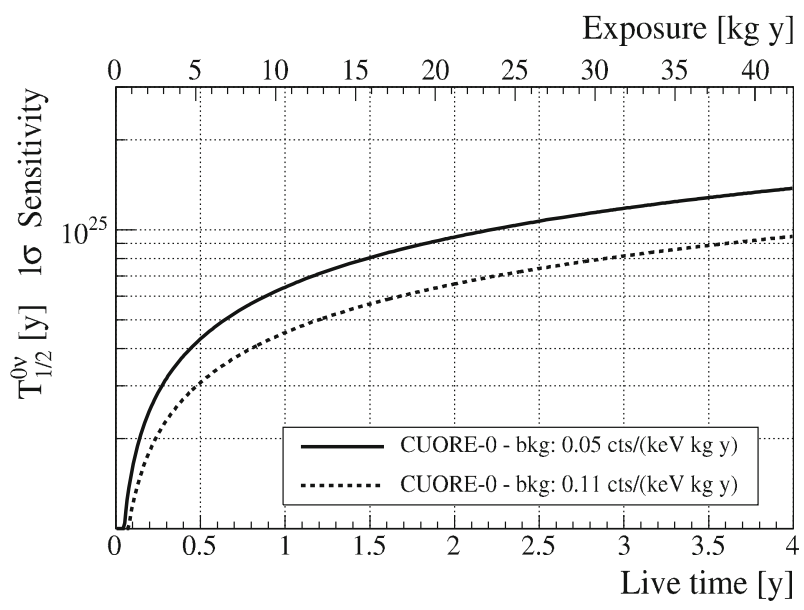

Fig. 3 CUORE- 0 sensitivity at $1 \sigma$ for two different values of the background rate in the ROI: 0.05 counts $/ \mathrm{keV} / \mathrm{kg} / \mathrm{y}$ (solid line) and 0.11 counts/keV/kg/y (dotted line). The CUORE-0 background is expected to fall within this range.

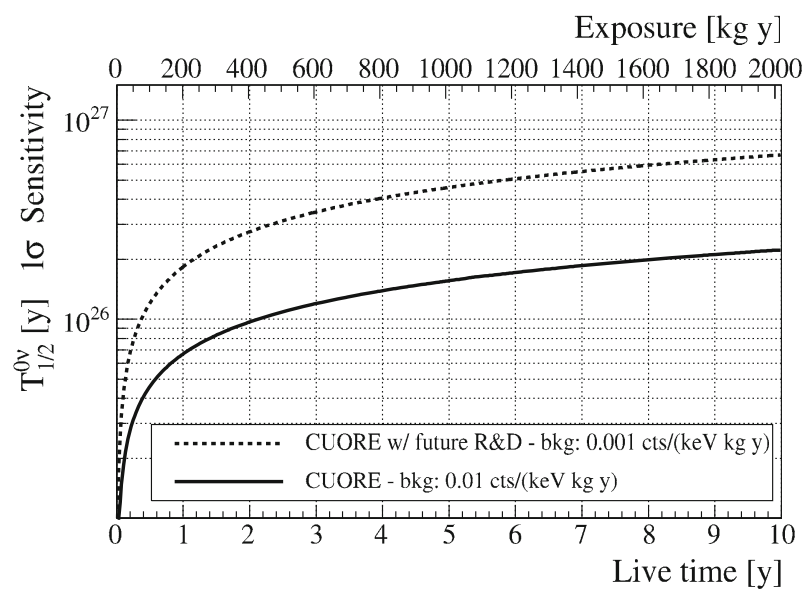

Fig. 4 Expected sensitivity for CUORE as a function of live time and exposure.

R\&D bolometric measurements in which the contamination of the crystals and copper surfaces was reduced by means of new surface treatments [7], [8]).

A plot of the expected $1 \sigma$ sensitivity of CUORE- 0 as a function of live time in these two bounding cases is shown in Fig. 3.

\section{CUORE Status and Sensitivity}

The commissioning of the CUORE cryostat started during summer 2012 and is currently ongoing. All the crystal have been delivered to LNGS and the first six towers of the CUORE array have been assembled.The installation and cool down of the CUORE 
detector and the start of detector operations are foreseen for the end of 2014. A plot of the CUORE experimental sensitivity as a function of the live time and exposure is shown in Fig. 4. Assuming a background of 0.01 counts $/ \mathrm{keV} / \mathrm{kg} / \mathrm{y}$ and five years of live time, the CUORE half-life sensitivities at $1 \sigma$ would be $1.6 \times 10^{26}$ years. This would mean sensitivity to an effective Majorana neutrino mass in the range 51 and $133 \mathrm{meV}$ (90\% C.L.) depending on nuclear matrix elements considered. The predicted sensitivity of CUORE would allow the investigation of the upper region of the effective Majorana neutrino mass phase space for the inverted hierarchy of neutrino masses.

Acknowledgments The CUORE Collaboration thanks the Directors and Staff of the Laboratori Nazionali del Gran Sasso and the technical staff of our Laboratories. This work was supported by the Istituto Nazionale di Fisica Nucleare (INFN); the Director, Office of Science, of the U.S. Department of Energy under Contract Nos. DE-AC02-05CH11231 and DE-AC52-07NA27344; the DOE Office of Nuclear Physics under Contract Nos. DE-FG02-08ER41551 and DEFG03-00ER41138; the National Science Foundation under Grant Nos. NSF-PHY-0605119, NSF-PHY-0500337, NSF-PHY-0855314, and NSF-PHY-0902171; the Alfred P. Sloan Foundation; and the University of Wisconsin Foundation.

\section{References}

1. A. Strumia and F.Vissani, arXiv:hep-ph/0606054v3 (2010).

2. M. Redshaw et al., Phys. Rev. Lett. 102, 212502 (2009)

3. E. Fiorini, T. Niinikoski, Nucl. Instrum. Meth. A 224, 83 (1984)

4. A. Alessandrello et al., Phys. Lett. B 433, 156-162 (1998)

5. E. Andreotti et al., Astropart. Phys. 34, 822-831 (2011)

6. C. Bucci et al., Eur. Phys. J. A 41, 155 (2009)

7. F. Alessandria et al., AstroPart. Phys. 45, 13-22 (2013)

8. F. Alessandria et al., Astropart. Phys. 35, 839 (2012)

9. M. Clemenza et al., Eur. Phys. J. C 71, 1805 (2011) 